\title{
Descartes's Independence Conception of Substance and His Separability Argument for Substance Dualism
}

\author{
Robert K. Garcia \\ Texas AEM University
}

robertkgarcia@gmail.com

www.robertkgarcia.com

\section{Request from the author:}

If you would be so kind, please send me a quick email if ...

- you are reading this for a university or college course, or

- you are citing this in your own work.

It is rewarding to know how my work is being used, especially if it has been adopted as required or recommended reading.

Thank you.

\section{Citation Information:}

Garcia, R. (2014). "Descartes's Independence Conception of Substance and His Separability Argument for Substance Dualism," Journal of Philosophical Research 39, 165-190. 


\title{
DESCARTES'S INDEPENDENCE CONCEPTION OF SUBSTANCE AND HIS SEPARABILITY ARGUMENT FOR SUBSTANCE DUALISM
}

\author{
ROBERT K. GARCIA \\ TEXAS A\&M UNIVERSITY
}

\begin{abstract}
I critically examine the view that Descartes's independence conception (IC) of substance plays a crucial role in his "separability argument" for substance dualism. I argue that IC is a poisoned chalice. I do so by considering how an IC-based separability argument fares on two different ways of thinking about principal attributes. On the one hand, if we take principal attributes to be universals, then a separability argument that deploys IC establishes a version of dualism that is unacceptably strong. On the other hand, if we take principal attributes to be tropes, then IC introduces challenges that undermine the argument. This is partly because the assumption of tropes makes it possible to distinguish several versions of substance dualism, versions that differ with respect to their degree of generality. I argue that taking principal attributes to be tropes makes it challenging to establish any of these versions by way of an IC-based separability argument. I conclude the paper by suggesting a way forward for the proponent of the separability argument.
\end{abstract}

$\mathrm{I}_{\mathrm{t}}$ is widely held that Descartes is a substance dualist, one who holds that there are exactly two kinds of finite substance — mind and body — and that no mind could have a material property and no body could have a mental property. ${ }^{1}$ Descartes's chief argument for substance dualism is the so-called "separability argument" found in the Sixth Meditation. There is considerable dispute over the background assumptions and exact structure of this argument. ${ }^{2}$ Because Descartes had more than one conception of substance, an important question concerns whether the separability argument relies crucially on one or more of these conceptions. On one conception, a substance is an independent entity, whereas, on another conception, a substance is a subject of properties. Philosophers 
such as Jonathan Bennett and Louis Loeb have argued for the preeminence of the subject conception in Descartes's metaphysics. Others, such as Gonzalo RodriguezPereyra, assign that status to the independence conception. ${ }^{3}$

In this paper I critically examine the view that the independence conception (IC) plays a crucial role in the separability argument and in Descartes's metaphysics more generally. I do so by considering how a separability argument that assumes IC fares on two different ways of thinking about the metaphysical status of principal attributes-as universals or tropes. (I gloss the universal/trope distinction below.) Although it is unlikely that Descartes took principal attributes to be universals, it will be instructive briefly to consider how the separability argument fares if we construe principal attributes in this way. Doing so will provide a useful foil for a separability argument on which principal attributes are taken to be tropes. Along the way, I will draw from the contemporary discussion of tropes to clarify the prospects of the separability argument.

My principal claims are the following. With respect to the separability argument, the independence conception of substance is a poisoned chalice. If we take principal attributes to be universals, then a separability argument that deploys IC establishes a version of dualism that is unacceptably strong. Alternatively, if we take principal attributes to be tropes, then IC introduces challenges that threaten to undermine the argument. As we will see, this is partly because the assumption of tropes allows us to distinguish between several versions of substance dualism, versions that differ with respect to their degree of generality. I will argue that the assumption of tropes makes it challenging to establish any version by way of an IC-based separability argument. A tropist separability argument that aims at securing a general thesis — one ranging over all minds and bodies_-must be expanded so as to include a generalizing step. Motivating this step, however, is made difficult by IC, the very principle alleged to be crucial for the separability argument. Moreover, if principal attributes are tropes and not universals, then IC does not imply the Cartesian doctrine that a substance and its principal attribute are (merely) conceptually distinct. Thus, if principal attributes are tropes, IC does not supply the separability argument with a crucial premise. Indeed, without this doctrine, the argument does not secure even the weakest version of substance dualism. In sum, I argue that if the separability argument for substance dualism is to be understood as requiring an implicit appeal to $\mathrm{IC}$, then it is either unacceptably strong (where principal attributes are universals) or not strong enough (where principal attributes are tropes). I conclude the paper by suggesting a way forward for the proponent of the separability argument. At the end of section one I will introduce my theses in sharper detail, against the backdrop of a reconstruction of the separability argument.

\section{A PROVISIONAL SEPARABILITY ARGUMENT}

We find Descartes's original separability argument in the following passage from the Sixth Meditation:

First, I know that everything which I clearly and distinctly understand is capable of being created by God so as to correspond exactly with my understanding of 
it. Hence the fact that I can clearly and distinctly understand one thing apart from another is enough to make me certain that the two things are distinct, since they are capable of being separated, at least by God. The question of what kind of power is required to bring about such a separation does not affect the judgment that the two things are distinct. Thus, simply by knowing that I exist and seeing at the same time that absolutely nothing else belongs to my nature or essence except that I am a thinking thing, I can infer correctly that my essence consists solely in the fact that I am a thinking thing. It is true that I may have (or, to anticipate, that I certainly have) a body that is very closely joined to me. But nevertheless, on the one hand I have a clear and distinct idea of myself, in so far as I am simply a thinking, non-extended thing; and on the other hand I have a distinct idea of body, in so far as this is simply an extended, non-thinking thing. And accordingly, it is certain that I am really distinct from my body, and can exist without it. (CSM II, 54; AT VII, 78) ${ }^{4}$

Before presenting a reconstruction of the argument, I would like to make a few preliminary comments concerning how my aims in this paper bear on how I structure the separability argument. Under consideration is the two-fold thesis that IC is central to Descartes's metaphysics and that IC's centrality is evinced by the fact that it plays a crucial role in the separability argument. This thesis has recently been defended by Rodriguez-Pereyra, who has proposed a formulation of the separability argument that illuminates the need for IC to play a role. I find the basic outline of his reconstruction to be faithful to Descartes and interesting in its own right. I also wish to use a formulation of the separability argument that is charitable to the thesis under consideration. Accordingly, I will present a formulation that is essentially based on Rodriguez-Pereyra's. Nevertheless, I have made some changes and additions to his formulation in order to facilitate the discussion of two interrelated issues.

The first issue concerns the intended generality of the separability argument's conclusion, substance dualism. Although the Meditations are written from a first-person perspective, there are reasons for thinking that Descartes aimed at a fully general conclusion - a conclusion not only about the meditator's mind and body but about every mind and body, ${ }^{5}$ and perhaps even about every possible mind and body. ${ }^{6}$ Although several commentators have noted that the argument requires such a "generalizing move," most seem to think that the generalization is unproblematic. ${ }^{7}$ However, I think the generalizing step deserves a more careful look. So in order to let the step stand on its own, my initial formulation of the argument begins in the first-person and concludes with a generalizing step. Later I will consider what sorts of premises (if any) must be added in order to justify the generalizing step.

The second issue concerns the ontological status of the principal attributes referred to in the argument. I wish to consider how the argument fares on two fundamentally different ways of thinking about principal attributes-as either universals or tropes. Accordingly, I initially formulate the argument in a way that is ambiguous with respect to the universal/trope distinction. I then go on to discuss the merits of both a realist and a tropist interpretation of the argument, focusing on the latter. 
These two issues are interrelated. As I will argue, whether the generalizing step is problematic or not depends, in part, on whether principal attributes are taken to be universals or tropes.

My final preliminary comment concerns how a formulation of the separability argument ought to represent the Sixth Meditation's notion of "clear and distinct understanding." Here I follow others in amplifying this phrase in light of what Descartes says in his Replies. ${ }^{8}$ In response to an objection from Arnauld, Descartes seems to grant that from the fact that one can clearly and distinctly understand $a$ apart from $b$, it doesn't follow that $a$ can exist apart from $b$ (AT VII, 227). But Descartes goes on to argue that if one had a complete understanding of $a$ apart from $b$, then it would follow that $a$ can exist apart from $b$. In fact, it would follow that $a$ is a substance (AT VII, 222). In light of this, I accept Rodriguez-Pereyra's proposal that we formulate the separability argument in terms of "clear, distinct, and complete understanding," and, more specifically, as deploying premise (1) below. ${ }^{9}$ Others may wish to resist this premise, but as my interests lie elsewhere, I will grant it in what follows.

So much for preliminary comments. In the following reconstruction of the separability argument, let $M_{1}$ stand for my (or the meditator's) mind, $B_{1}$ stand for my (or the meditator's) body, T stand for a principal attribute of thought, and E stand for a principal attribute of extension.

(1) If I clearly, distinctly, and completely understand $a$ apart from $b$, then $a$ is a substance and it is possible that $a$ exists without $b$.

(2) If it is possible that $a$ exists without $b$, then $a \neq b$ ( $a$ and $b$ are numerically distinct).

(3) I clearly, distinctly, and completely understand my mind $\left(M_{1}\right)$ which has an attribute of thought $(\mathrm{T})$, apart from my body $\left(B_{1}\right)$ which has an attribute of extension (E); and I clearly, distinctly, and completely understand my body $\left(B_{1}\right)$ which has an attribute of extension $(\mathrm{E})$, apart from my mind $\left(M_{1}\right)$ which has an attribute of thought $(\mathrm{T}) .{ }^{10}$

(4) Thus, $M_{1}$ is a substance, and it is possible that it exists without $B_{1}$, and $B_{1}$ is a substance, and it is possible that it exists without $M_{1}$. [by 1,3 ]

(5) Thus, my mind and my body are numerically distinct, $M_{1} \neq B_{1}$. [by 2, 4]

(6) Thus, it is possible that one thing has T but not E, and it is possible that another thing has $\mathrm{E}$ but not $\mathrm{T}$. [by 3, 5]

(7) If it is possible that one thing has property $\mathrm{F}$ but not $\mathrm{G}$ and another thing has property $\mathrm{G}$ but not $\mathrm{F}$, then $\mathrm{F} \neq \mathrm{G}$. [premise]

(8) Thus, principal attribute of thought $\mathrm{T}$ is distinct from principal attribute of extension $\mathrm{E} ; \mathrm{T} \neq \mathrm{E}$. [6, 7]

(9) Thus, for all $\mathrm{F}$ and all $\mathrm{G}$, if $\mathrm{F}$ is a principal attribute of thought and $\mathrm{G}$ is a principal attribute of extension, then $\mathrm{F} \neq \mathrm{G}$. [generalizing step] 
With this formulation of the separability argument in place, we will now consider the general thesis that IC is crucial for the success of the argument. If this general thesis is correct, then, as it stands, the above argument fails to establish substance dualism, but would succeed with the aid of IC.

It certainly seems that the above argument fails to establish dualism. As Rodriguez-Pereyra points out, the argument faces an immediate objection, the substance of which was raised by Regius, a contemporary of Descartes:

Regius's Objection: The attributes of extension and thought are not opposites but merely different, so an argument for their difference is not an argument for their opposition. ${ }^{11}$

In other words, the above argument isn't an argument for substance dualism, but only for the real distinction and property dualism theses. These three theses are distinct:

Real Distinction: Any mind and any body are non-identical substances (generalizing from (5) above).

Property Dualism: For all $\mathrm{F}$ and all $\mathrm{G}$, if $\mathrm{F}$ is a principal attribute of thought and $\mathrm{G}$ is a principal attribute of extension, then $\mathrm{F} \neq \mathrm{G}$. More generally, any mental property and any material property are non-identical. ${ }^{12}$

Substance Dualism: Any substance with a mental property lacks a material property and any substance with a material property lacks a mental property. ${ }^{13}$

According to Regius's Objection, the argument may show that mental and physical properties can be separate and thus be non-identical (thereby establishing property dualism), but it doesn't show that they must be separate. In other words, at best it shows that something can have mental properties without physical properties, and that something can have physical properties without mental properties. But it doesn't show that mental and physical properties are such that nothing can have both. Thus, while the argument establishes property dualism, it does not establish substance dualism.

According to Rodriguez-Pereyra, Descartes's answer to this objection is found in his general views about substance. Importantly, the crucial aid comes from Descartes's independence conception (IC) and not his subject conception. Roughly, the latter conception takes a substance to be a basic subject of properties (CSM II, 114; AT VII, 161). On the former conception, a substance is an independent entity. Descartes writes:

By substance we can understand nothing other than a thing which exists in such a way as to depend on no other thing for its existence. And there is only one substance which can be understood to depend on no other thing whatsoever, namely God. In the case of all other substances, we perceive that they can exist only with the help of God's concurrence. Hence the term "substance" does not apply univocally. (CSM I, 210; AT VIIIA, 24)

We can spell this out more precisely in terms of what I will call token dependence. (In what follows, I use "entity" as the most general count noun, a catch-all, categoryneutral term for any kind of thing.) An entity $a$ is token dependent on $b$ if and only 
if, necessarily, $a$ exists only if $b$ exists. Crucially, this is called token dependence because if $a$ is token dependent on $b$, then $a$ 's existence condition cannot be satisfied by the existence of some third entity $c$, regardless of how similar $c$ is to $b$ (even if $b$ and $c$ are, say, intrinsic duplicates). The notion of token dependence can be used to state Descartes's independence conception more precisely:

IC: $x$ is a substance if and only if there is no (distinct) $y$ such that $y \neq \operatorname{God}$ and $x$ is token dependent on $y$.

Notice that the sort of dependence that disqualifies something from being a substance is dependence on a (distinct) specific or token (non-divine) entity. It is consistent with $x$ 's being a substance that $x$ cannot exist without some type of entity or other. ${ }^{14}$ For example, minds and bodies are supposed to satisfy IC in that while a mind (or body) requires some mode of thought (extension) or other to exist, it doesn't require any given or token mode. A body requires some shape or other to exist, but not necessarily this specific or token shape.

According to Rodriguez-Pereyra, IC provides Descartes with something the subject conception of substance cannot-a way to respond to Regius's Objection. This is because only IC secures the Cartesian doctrine that there is a mere conceptual distinction between a principal attribute and the substance that has it (CSM I, 213-214; AT VIIIA, 28-31). According to this doctrine, a substance and its principal attribute are not distinct entities. Rather, we have two different concepts for thinking about the very same entity (Rodriguez-Pereyra 2008, 77-78). Thus, we may put the conceptual distinction principle as follows:

DP: A substance $x$ has principal attribute $\mathrm{F}$ if and only if $x=\mathrm{F}$.

This principle may be derived from IC in the following way. If IC is true, then God is the only distinct entity on which a substance can be token dependent. Thus, if substance $a$ is token dependent on some entity $b$, then either $a=b$ or $\operatorname{God}=b$. But a substance's principal attribute is a specific entity without which the substance cannot exist (a principal attribute constitutes the essence of a substance). Thus (on pain of identifying each principal attribute with God) ${ }^{15}$ a substance and its principal attribute are not distinct entities (DP). In other words, IC has "the consequence that no attribute of a substance is an entity other than the substance in question. But from this it follows that ... only a conceptual distinction can obtain between a substance and its attribute" (Rodriguez-Pereyra 2008, 82). ${ }^{16}$ In other words, IC implies DP.

$\mathrm{DP}$, in turn, entails a further principle (DP*), which enables the separability argument to secure not just property dualism but substance dualism (and thereby to overcome Regius's Objection). In particular, DP* allows the argument to infer the incompossibility of two principal attributes from their non-identity. The steps from DP to DP* are the following:

(D1) DP: A substance $x$ has principal attribute $\mathrm{F}$ if and only if $x=\mathrm{F}$.

(D2) Thus, a substance $x$ has principal attributes $\mathrm{F}$ and $\mathrm{G}$ only if $x=\mathrm{F}$ and $x=\mathrm{G}$.

(D3) Thus, a substance $x$ has principal attributes $\mathrm{F}$ and $\mathrm{G}$ only if $\mathrm{F}=\mathrm{G}$. 
(D4) Thus, if it is impossible that $\mathrm{F}=\mathrm{G}$, then it is impossible that a substance $x$ have both $\mathrm{F}$ and $\mathrm{G}$.

(D5) Identity is necessary: if $\mathrm{F} \neq \mathrm{G}$, then it is impossible that $\mathrm{F}=\mathrm{G} .{ }^{17}$

(D6) Thus, $\mathrm{DP}^{*}$ : if $\mathrm{F} \neq \mathrm{G}$, then it is impossible that a substance $x$ have both $\mathrm{F}$ and $\mathrm{G}$.

If $\mathrm{DP}^{*}$ is appended to the above separability argument, the intended conclusion can be secured. Here is the last premise from above, together with $\mathrm{DP}^{*}$ and the intended conclusion:

(9) Thus, for all $\mathrm{F}$ and all $\mathrm{G}$, if $\mathrm{F}$ is a principal attribute of thought and $\mathrm{G}$ is a principal attribute of extension, then $\mathrm{F} \neq \mathrm{G}$. [generalizing step]

(10) If $F \neq G$, then it is impossible that a substance $x$ have both $F$ and G. [DP*]

(11) Thus, for all F and all G, if F is a principal attribute of thought and G is a principal attribute of extension, then it is impossible that a substance $x$ have both $\mathrm{F}$ and $\mathrm{G}$. [9, 10]

(12) Thus, more generally, any substance with a mental property lacks a material property and any substance with a material property lacks a mental property. ${ }^{18}$

It seems, then, that IC plays the crucial role of enabling the separability argument to overcome Regius's Objection. By establishing the doctrine (DP) that there is only a conceptual distinction between a substance and its principal attribute, IC provides the separability argument with $\mathrm{DP}^{*}$, the premise needed to secure substance dualism and not merely property dualism. Given the centrality of substance dualism for Descartes, this constitutes a strong case for the overall importance of IC in Descartes's metaphysics.

Below, however, I will argue that IC isn't as helpful as it seems - in fact, IC is a poisoned chalice. In part, this is because there are different ways of thinking about the metaphysical status of the principal attributes referred to in the separability argument. On the one hand, we can think of principal attributes as universals, which are unique and literally sharable properties. For example, if sphericalness is a universal, then qua sharable, sphericalness can be instantiated by (characterize, etc.) multiple objects at once. And, qua unique, there isn't a sphericalness other than the one that there is. (More about uniqueness below.) On the other hand, we can think of principal attributes as non-unique and non-sharable properties, what contemporary metaphysicians call tropes. ${ }^{19}$ Tropes are non-sharable in that it is impossible that any given trope be the property of more than one object (at once). ${ }^{20}$ Tropes are non-unique in that for any given trope $T$, it is possible that there be a distinct trope $T^{*}$ that is exactly similar to $T$. For example, there could have been (and typically are) distinct sphericalness tropes besides (or in addition to) any given sphericalness trope that there is. (More on this below.) Thus, the distinction between universals and tropes provides two different ways of understanding the above separability argument. 
The rest of the paper unfolds as follows. In the next section I will consider how an IC-based separability argument fares if we take principal attributes to be universals. (I should emphasize, however, that I do not attribute this argument to Descartes, who arguably did not countenance universals and took principal attributes to be something like tropes. $)^{21}$ In section III, I will consider how an IC-based separability argument fares if we take principal attributes to be tropes. In sum, I argue that if the separability argument for substance dualism is to be understood as requiring an implicit appeal to IC, then it is either unacceptably strong (where principal attributes are universals) or not strong enough (where principal attributes are tropes). I conclude the paper by suggesting a way forward for the proponent of the separability argument.

\section{A REALIST SEPARABILITY ARGUMENT}

We are now ready to consider how the above IC-based separability argument fares if we take principal attributes to be universals. We face an immediate problem, however. According to DP, a substance $x$ has principal attribute $\mathrm{F}$ if and only if $x=\mathrm{F}$. Thus, for any substances $x$ and $y, x$ and $y$ both have $\mathrm{F}$ if and only if $x=y$. In other words, DP entails that a principal attribute is non-sharable. Thus, in a separability argument that deploys DP, principal attributes must be understood to be non-shareable properties.

This leaves us with two general ways to understand principal attributes. On the one hand, we can take them to be tropes, which are non-sharable and non-unique properties. This way of understanding principal attributes will occupy us in the next section, where we will consider a tropist interpretation of the separability argument. On the other hand, we can take a principal attribute to be a non-sharable and unique property. This is a non-standard notion of a universal, what I will call a hybrid-universal. ${ }^{22}$ There is theoretical space for the notion of a hybrid-universal, and, as we will see, considering them will be instructive in that it will highlight the need for a generalizing step in a tropist version of the separability argument. Indeed, a central issue in what follows is whether and how a separability argument can secure a fully general conclusion.

The uniqueness of hybrid-universals allows the realist separability argument to straightforwardly secure a general conclusion. Uniqueness can be illustrated with an example. If sphericity property $\mathrm{F}$ is unique, then there is no sphericity property that is non-identical to $\mathrm{F}$. That is, if there is a sphericity property, then there is exactly one sphericity property. ${ }^{23}$ Now consider principal attributes $\mathrm{E}$ and $\mathrm{T}$, which we are taking to be hybrid-universals. Because E and T are unique, there isn't a principal attribute of thought that is non-identical to T, and there isn't a principal attribute of extension that is non-identical to E. This means that (8) straightforwardly entails (9). Thus, if principal attributes are hybrid-universals then the generalizing step of the separability argument is entirely unproblematic, even trivial. It does not require any additional argumentation or undergirding. In sum, a realist separability argument is able to secure substance dualism, thus overcoming Regius's Objection. 
It should be obvious, however, that taking principal attributes to be unique also saddles the separability argument with an unacceptable cost-its conclusion is too strong. If principal attributes are unique, then there is, at most, exactly one principal attribute of thought, and, at most, exactly one principal attribute of extension. However, according to DP, a substance has a principal attribute if and only if it is identical to that attribute. Thus, given uniqueness and DP, it follows that there is no more than one mind and no more than one body. More generally, the argument establishes not only substance dualism, but what I will call hyper substance dualism or HSD. The latter is substance dualism plus the following two theses:

One-Mind: It is impossible that there be more than one mind.

One-Body: It is impossible that there be more than one body.

As I will now argue, HSD is unacceptably strong and so it is highly desirable, if not necessary, that an argument for substance dualism not entail HSD. This is especially true if the argument is to be attributed to Descartes.

Clearly, Descartes was committed to the existence of more than one mind. And the coexistence of multiple minds is independently plausible, so I will assume it in what follows. It is less clear whether Descartes was committed to the existence of more than one body. ${ }^{24}$ Presumably, however, it would be especially controversial to attribute to him the view that there couldn't have been more than one body. So the realist separability argument seems to prove too much. The upshot is that Descartes's argument needs to be interpreted in such a way as to avoid the unacceptable components of HSD. (Note that avoiding a commitment to One-Body does not involve entailing its falsity, so the strategy is compatible with affirming One-Body.)

As we've seen, the inordinate strength of the realist separability argument stems from both DP and the assumption that principal attributes are unique. According to $\mathrm{DP}$, it is possible that there is more than one mind if and only if it is possible that there is more than one principal attribute of thought. But if principal attributes are unique, then it is not possible that there is more than one attribute of thought. Thus, to avoid HSD a proponent of the separability argument needs to either reject DP or deny that principal attributes are unique.

I will adopt the latter strategy in what follows. I do so because my general aim is to assess the thesis that IC plays a role in the separability argument and does so in virtue of providing the argument with DP (and thus DP*). Accordingly, I will leave DP in place and consider how the argument fares on the assumption that principal attributes are non-unique. As noted above, however, DP entails that principal attributes are non-sharable. Thus, I will now proceed on the assumption that principal attributes are neither unique nor sharable - that is, that they are tropes. An additional reason for construing principal attributes as tropes is that Descartes clearly construed modes as something like tropes. Indeed, Descartes's modes aren't significantly theoretically different from the tropes of many contemporary trope theorists. ${ }^{25}$ For example, Descartes held not only that a given mode is not possibly the mode of more than one substance (a mode is a non-sharable property), a mode 
is not possibly the mode of a substance other than the very one of which it is a mode (a mode is a non-transferable property). ${ }^{26}$

Not only is it plausible to construe Descartes's principal attributes as tropes, doing so precludes the commitment to HSD. For consider: If principal attributes are tropes then their non-uniqueness entails that it is possible that there be numerous numerically distinct attributes of extension and thought. And because tropes are non-sharable, if there are two minds, then there are two numerically distinct attributes of thought. In what follows, this will be represented by the use of subscripts when referring to attributes. For example, we might say that Jack's mind has $\mathrm{T}_{5}$, while Jill's mind has $\mathrm{T}_{7}$, where $\mathrm{T}_{5}$ and $\mathrm{T}_{7}$ are distinct principal attributes of thought.

Nevertheless, although taking principal attributes to be tropes obviates a commitment to HSD, it also raises new challenges for the separability argument. In part, this is because the assumption of tropes allows us to distinguish between several versions of substance dualism, versions that differ with respect to their degree of generality. Most important will be the distinction between what I will call local and minimal substance dualism. I will argue that the assumption of tropes makes it challenging to establish any version by way of a separability argument. My aim is to bear this out as follows. In the next section, I spell out different versions of substance dualism and argue that a tropist separability argument that retains the original aim-to secure at least a locally general version-must include a nontrivial generalizing step. In section IV I argue that motivating this generalizing step is actually undermined by IC, the very principle alleged to be crucial for the separability argument. IC thereby presents a challenge for local (and stronger versions of) substance dualism. In section V I argue that if principal attributes are tropes and not universals, then IC does not imply DP, and so IC does not provide the argument with the premise required to establish even minimal substance dualism, the weakest version. In sum, I argue that if the separability argument for substance dualism is to be understood as requiring an implicit appeal to IC, then it is either unacceptably strong (where principal attributes are hybrid-universals) or not strong enough (where principal attributes are tropes).

\section{A TROPIST SEPARABILITY ARGUMENT}

The assumption that principal attributes are tropes has implications for what it takes for a separability argument to succeed. I'll note these implications before offering a formulation of a tropist separability argument.

If principal attributes are tropes and not universals, then it is false that there is, at most, one attribute of thought. Rather, if principal attributes are tropes, then there is a numerically distinct attribute of thought for every mind that there is, and there could have been distinct attributes of thought (extension) besides (or in addition to) the attributes that there are. For example, the meditator's attribute of thought is distinct from any other attribute of thought that there is. Thus, within the context of the separability argument, if principal attributes are tropes, then strictly speaking it is a mistake to refer to the attribute of thought or the attribute 
of extension. Rather, we may refer to this or that attribute of extension, and we may use subscripts to represent their numerical distinctness (e.g., $\mathrm{E}_{1}, \mathrm{E}_{2}$, etc.). This language will be adopted in what follows.

Furthermore, if principal attributes are tropes then it is possible to distinguish between different versions of substance dualism, versions that vary with respect to their strength. This is important because whether a separability argument is successful depends, in part, on which version of substance dualism the argument is supposed to establish.

Some have suggested that Descartes's separability argument can (or perhaps should) be interpreted as aiming at the following fully general, or global thesis: ${ }^{27}$

Global Substance Dualism (GSD): Necessarily, for any principal attribute of thought $\mathrm{T}$ and any principal attribute of extension $\mathrm{E}$, no substance has both $\mathrm{T}$ and $\mathrm{E}$.

Others take Descartes to aim only at a weaker version of substance dualism, the sort presented earlier in this paper. ${ }^{28}$ There I presented a definition that lacks the modal qualifier 'necessarily.' I will call this the local version of substance dualism, and restate it as follows:

Local Substance Dualism (LSD): For any principal attribute of thought $\mathrm{T}$ and any principal attribute of extension $\mathrm{E}$, no substance has both $\mathrm{T}$ and $\mathrm{E}$.

It seems clear that Descartes intended to establish at least LSD. It is less clear whether he also aimed at GSD. Although I'm inclined to read him as aiming for the stronger thesis, in what follows I will assume that the separability argument aims for no more than LSD. The challenges I pose will arise, a fortiori, for a separability argument that also aims to secure GSD. ${ }^{29}$

LSD, however, is not the weakest version of substance dualism. The assumption of tropes allows us to identify a distinct minimalist version:

Minimal Substance Dualism (MSD): There is a principal attribute of thought $\mathrm{T}$ such that for any principal attribute of extension $\mathrm{E}^{*}$, no substance has both $\mathrm{T}$ and $\mathrm{E}^{*}$. And, there is a principal attribute of extension $\mathrm{E}$ such that for any principal attribute of thought $\mathrm{T}^{*}$, no substance has both $\mathrm{E}$ and $\mathrm{T}^{*}{ }^{30}$

The difference in generality between LSD and MSD is important because it represents the work that a generalizing step must do in a tropist separability argument. The above realist separability argument secures LSD (premise (11) above) by way of a trivial generalizing step_one that needs no additional argumentation or support. As I argue below, the tropist separability argument does not secure LSD so easily. Without a non-trivial generalizing step — one that needs additional support—a tropist separability argument fails to secure LSD. It seems, however, that the argument we find in the Sixth Meditation is an argument for a general conclusion, a tropist version of which would correspond to LSD (if not GSD). ${ }^{31}$ Thus, a faithful tropist reconstruction of the argument will aim at securing LSD and not merely MSD. Thus, unlike its realist counterpart, the generalizing step in a tropist separability argument will require additional support. (These points notwithstanding, in the 
final section I will briefly consider whether an argument that aims at only minimal dualism might suffice to achieve Descartes's more general aims in the Meditations.)

Recall that we are considering the general thesis that IC plays a crucial role in the argument by securing DP* (by way of DP). To reflect our assumption that principal attributes are tropes, the language of DP and DP* may be improved as follows:

DP: A substance $x$ has principal attribute $\mathrm{F}_{1}$ if and only if $x=\mathrm{F}_{1}$.

DP*: If $\mathrm{F}_{1} \neq \mathrm{G}_{1}$, then it is impossible that a substance $\mathrm{x}$ have both $\mathrm{F}_{1}$ and $\mathrm{G}_{1}$.

Having noted some of the relevant implications for taking principal attributes to be tropes, I will now consider an IC-based tropist separability argument. I'll begin by formulating the argument without a generalizing step. This will highlight the need for additional argumentation to secure LSD. I'll then consider possible ways to underwrite a generalizing step. Here is the tropist separability argument:

(1) If I clearly, distinctly, and completely understand $a$ apart from $b$, then $a$ is a substance and it is possible that $a$ exists without $b$.

(2) If it is possible that $a$ exists without $b$, then $a \neq b$ ( $a$ and $b$ are numerically distinct).

(3) I clearly, distinctly, and completely understand this $\left(\mathrm{M}_{1}\right)$ which has an attribute of thought $\left(\mathrm{T}_{1}\right)$ apart from that $\left(\mathrm{B}_{1}\right)$ which has an attribute of extension $\left(\mathrm{E}_{1}\right)$, and I clearly, distinctly, and completely understand that $\left(\mathrm{B}_{1}\right)$ which has an attribute of extension $\left(\mathrm{E}_{1}\right)$ apart from this $\left(\mathrm{M}_{1}\right)$ which has an attribute of thought $\left(\mathrm{T}_{1}\right)$.

(4) Thus, $M_{1}$ is a substance, and it is possible that it exists without $B_{1}$, and $B_{1}$ is a substance, and it is possible that it exists without $M_{1} \cdot[1,3]$

(5) Thus, my mind is numerically distinct from my body, $M_{1} \neq B_{1}$. [2, 4]

(6) Thus, it is possible that one thing has $\mathrm{T}_{1}$ but not $\mathrm{E}_{1}$, and it is possible that another thing has $\mathrm{E}_{1}$ but not $\mathrm{T}_{1}$. [by 3,5$]$

(7) If it is possible that one thing have trope $\mathrm{F}_{1}$ but not $\mathrm{G}_{1}$ and another thing have trope $\mathrm{G}_{1}$ but not $\mathrm{F}_{1}$, then $\mathrm{F}_{1} \neq \mathrm{G}_{1}$. [premise]

(8) Thus, my principal attribute of thought is distinct from my principal attribute of extension, $\mathrm{T}_{1} \neq \mathrm{E}_{1}$. [6, 7]

(9) If $\mathrm{T}_{1} \neq \mathrm{E}_{1}$, then it is impossible that a substance $x$ have both $\mathrm{T}_{1}$ and $\mathrm{E}_{1}$ [DP*].

(10) Thus, it is impossible that something have both $\mathrm{E}_{1}$ and $\mathrm{T}_{1}$; i.e., $\mathrm{E}_{1}$ and $\mathrm{T}_{1}$ are incompossible tropes. [8,9]

The first thing to note is that, as it stands, it is unclear whether the tropist argument suffices to secure even the weakest, minimalist version of dualism (MSD). If principal attributes are tropes, then there can be other principal attributes of extension besides $\mathrm{E}_{1}$. Thus, in order for the argument to establish MSD, we must 
assume that the substance that has $\mathrm{T}_{1}$ lacks not only $\mathrm{E}_{1}$ but any other principal attribute of extension.

Nevertheless, it is reasonable to think that this assumption is secured by Descartes's notion of a "complete understanding" as represented in the first three premises. (Recall that I am following others in interpreting Descartes as appealing to a clear, distinct and complete understanding, rather than to merely a clear and distinct one.) On this reading of Descartes, the meditator not only clearly and distinctly perceives her mind apart from her body, she also has a complete idea of her mind (and of her body). ${ }^{32}$ Presumably, if I have a complete understanding of my mind (in Descartes's sense of “complete"), I will know whether or not my mind has an attribute of extension. As my interest in this paper lies elsewhere, I am happy to grant that the mediator knows this in virtue of clearly, distinctly, and completely understanding her mind. If we grant this, then the above argument establishes MSD.

However, even if the above argument establishes MSD, its conclusion is not general enough to secure LSD. This is because the conclusion is consistent with there being a principal attribute of thought non-identical to $\mathrm{T}_{1}$ (call it $\mathrm{T}_{2}$ ), a principal attribute of extension non-identical to $\mathrm{E}_{1}$ (call it $\mathrm{E}_{2}$ ), and a substance that has both $\mathrm{T}_{2}$ and $\mathrm{E}_{2}$. In other words, the conclusion is consistent with the denial of LSD. To establish LSD, it is not enough for the conclusion to range over the tropes of which the meditator is directly aware (i.e., $\mathrm{T}_{1}$ and $\mathrm{E}_{1}$ ). Rather, the conclusion needs to range over all thought and extension tropes. Otherwise, it would be consistent with the denial of LSD. (And if the argument is supposed to establish GSD, then the conclusion needs to range over all possible thought and extension tropes.) Thus, in order to secure LSD a tropist separability argument must include an additional generalizing step from (a) the incompossibility of the thought trope and extension trope with which the meditator is acquainted to (b) the incompossibility of all thought and extension tropes. Supplemented and refitted with such a step, the above argument would secure LSD. There seem to be two general strategies for supplying a generalizing step. Each strategy puts a step at a different place in the argument, but each involves the addition of a conditional premise.

The First Strategy is to add a conditional immediately after premise (3), which will allow the remaining premises to range over not just $\mathrm{T}_{1}$ and $\mathrm{E}_{1}$ but all attributes of thought and extension. The conclusion would thus be a statement of LSD. On this strategy, we add the following conditional premise after (3):

C1: If (a) I clearly, distinctly, and completely understand this $\left(\mathrm{M}_{1}\right)$, which has an attribute of thought $\left(\mathrm{T}_{1}\right)$, apart from that $\left(\mathrm{B}_{1}\right)$, which has an attribute of extension $\left(\mathrm{E}_{1}\right)$, and I clearly, distinctly, and completely understand that $\left(\mathrm{B}_{1}\right)$, which has an attribute of extension $\left(\mathrm{E}_{1}\right)$, apart from this $\left(\mathrm{M}_{1}\right)$, which has an attribute of thought $\left(\mathrm{T}_{1}\right)$, then (b) for any attribute of thought $\mathrm{T}$ and any attribute of extension E, I could clearly, distinctly, and completely understand the bearer of $\mathrm{T}$ apart from the bearer of E, and I could clearly, distinctly, and completely understand the bearer of $\mathrm{E}$ apart from the bearer of $\mathrm{T}$.

An alternative, Second Strategy, is to append the following conditional to the end of the argument: 
C2: If it is impossible that something have both $\mathrm{E}_{1}$ and $\mathrm{T}_{1}\left(\mathrm{E}_{1}\right.$ and $\mathrm{T}_{1}$ are incompossible), then for any attribute of extension $\mathrm{E}^{*}$ and any attribute of thought $\mathrm{T}^{*}$, it is impossible that something have both $\mathrm{E}^{*}$ and $\mathrm{T}^{*}\left(\mathrm{E}^{*}\right.$ and $\mathrm{T}^{*}$ are incompossible).

The success of either strategy requires two moves. The first move is to identify some relationship $\mathrm{R}$, such that for any thought trope $\mathrm{T}^{*}$ and any extension trope $\mathrm{E}^{*}$, it is plausible both that $\mathrm{T}_{1}$ and $\mathrm{T}^{*}$ stand in $\mathrm{R}$, and that $\mathrm{E}_{1}$ and $\mathrm{E}^{*}$ stand in $\mathrm{R}$. The second move is to motivate at least one of the following claims:

S1: [on the First Strategy] If ( $\mathrm{T}_{1}$ and $\mathrm{T}^{*}$ stand in $\mathrm{R}$, and $\mathrm{E}_{1}$ and $\mathrm{E}^{*}$ stand in $\mathrm{R})$, then $\mathrm{C} 1$ is true.

Or:

S2: [on the Second Strategy] If ( $\mathrm{T}_{1}$ and $\mathrm{T}^{*}$ stand in $\mathrm{R}$, and $\mathrm{E}_{1}$ and $\mathrm{E}^{*}$ stand in $\mathrm{R})$, then $\mathrm{C} 2$ is true.

This is a good place to take stock. What we've seen is that to avoid an inordinately strong conclusion (i.e., HSD), an IC-based separability argument must take principal attributes to be tropes and not universals. This, in turn, requires the argument to include a generalizing step, and this step requires motivating either S1 or S2. In the next two sections I will raise two challenges for the tropist separability argument. The first challenge concerns the plausibility of the generalizing step required to secure LSD. The second challenge is more fundamental and arguably more formidable. Recall that for an IC-based separability argument, IC plays the crucial role of providing DP* (by way of DP). The second challenge concerns the inference from IC to DP within a framework in which principal attributes are tropes.

\section{FIRST CHALLENGE: THE GENERALIZING STEP}

To secure LSD, a tropist separability argument requires a load-bearing generalizing step, and this can be provided by motivating either S1 or S2. In this section I will raise challenges for both strategies. But rather than proceed by considering each strategy at a time, I will consider how a few natural candidates for R fare with respect to both strategies.

As a first candidate for $\mathrm{R}$, consider identity. Although this is really a non-starter, it will prove instructive. Identity is a non-starter because in order to avoid HSD we are assuming that principal attributes are tropes. This assumption implies that there are or could be attributes of thought that are non-identical to $M_{1}$ (likewise for $\mathrm{E}_{1}$ ). Notice, however, that if $\mathrm{R}$ were identity, then the inferences represented by $\mathrm{C} 1$ and $\mathrm{C} 2$ would be trivial: in each, the antecedent and consequent would be equivalent. But because identity is not an option, the generalizing step is a nontrivial, substantive addition to the tropist separability argument. Thus, ruling out identity is instructive because regardless of which of the available candidates is $\mathrm{R}$, adding a generalizing step to the tropist separability argument will weaken it. In reformulating the argument so as to make it strong enough to establish LSD but not so strong as to entail HSD, we've ended up with a weaker argument than 
before. Just how much the generalizing step weakens the argument remains to be seen. In what follows I will mention several considerations that suggest that the step weakens the argument to a significant degree. These will not be anything close to knock-down arguments, but they indicate that the proponent of the tropist separability argument must shoulder a significant burden of proof. Of course, ultimately the reader will have to judge.

As a second candidate for $\mathrm{R}$, consider intersubstitutability. The idea is that a pair of tropes are intersubstitutable if and only if they could swap bearers without non-trivially changing those bearers. For any tropes $\mathrm{F}$ and $\mathrm{F}^{*}$, such that substance $a$ has $\mathrm{F}$ and $b$ has $\mathrm{F}^{*}, \mathrm{~F}$ and $\mathrm{F}^{*}$ are intersubstitutable if and only if (i) it is possible that $a$ has $\mathrm{F}^{*}$, (ii) it is possible that $b$ has $\mathrm{F}$, and (iii) there is only a trivial difference between having $F$ and having $F^{*}$. Roughly, between $a$ 's being $F$ and a's being $F^{*}$ there is a difference of thisness but not whatness. ${ }^{33}$ If two tropes are intersubstitutable, then we would expect them to be exactly similar with respect to their incompossibility profiles - that is, with respect to the tropes they are and are not compossible with. Thus, if the meditator's thought trope T1 is incompossible with her extension trope E1 and intersubstitutable with any possible thought trope $\mathrm{T}^{*}$, then $\mathrm{T}^{*}$ must also be incompossible with $\mathrm{E} 1$. More would have to be said, but we have the makings here of an argument from intersubstitutability to both S1 and S2. Thus, intersubstitutability is a natural and promising candidate for $\mathrm{R}$ and provides one strategy for defending the generalizing step. Unfortunately, however, this strategy is not available to Descartes.

As we will see, this is because the necessary conditions for intersubstitutability cannot be met if IC and/or DP are true. There are three necessary conditions for intersubstitutability. Crucial for the first two is the concept of token dependence, introduced above. (Recall that an entity $a$ is token dependent on $b$ if and only if, necessarily, $a$ exists only if $b$ exists.) First, $\mathrm{F}$ and $\mathrm{F}^{*}$ are intersubstitutable only if each is had contingently. Where substance $a$ has trope F, F is had contingently if and only if it is possible that $a$ exist but $\mathrm{F}$ not exist. That is, $\mathrm{F}$ is had contingently only if the substance that has F is not token dependent on F. Second, F and F* are intersubstitutable only if each is transferable. For any substance $a$ that has trope $\mathrm{F}, \mathrm{F}$ is transferable if and only if it possible that $\mathrm{F}$ be had by a substance other than a. Arguably, however, a trope is transferable only if it is not token dependent on its bearer. This is because taking a trope to be both transferable and token dependent leads to idiosyncratic and arguably unacceptable consequences concerning the ways in which substances affect and depend on each other. ${ }^{34}$ On pain of saddling the tropist separability argument with such consequences, I will assume that a trope is transferable only if it is not token dependent on its bearer. Third, $\mathrm{F}$ and $\mathrm{F}^{*}$ are intersubstitutable only if $\mathrm{F}$ and $\mathrm{F}^{*}$ are maximally similar such that there would be only a trivial difference (a mere difference in thisnesses) between a substance having $\mathrm{F}$ and that substance having $\mathrm{F}^{*} .35$

There are two reasons why an appeal to intersubstitutability is not available for Descartes. The first reason is that DP prevents the first two conditions from being satisfied. The second is that independently of DP, IC prevents the second condition from being satisfied. 
As to the first, DP blocks intersubstitutability because it entails that principal attributes are neither had contingently nor transferable. For example, suppose substance $a$ has principal attribute F. According to DP, $a=\mathrm{F}$. But F is had contingently only if it is possible that $a$ exist and not have F. Given DP, this means that $\mathrm{F}$ is had contingently only if it is possible that $a$ exist and $a \neq \mathrm{F}$. But identity is necessary, so because $\mathrm{a}=\mathrm{F}$, it is impossible that $a \neq \mathrm{F}$. Thus, DP entails that principal attributes are not had contingently. DP precludes transferability in a similar fashion. Attribute $\mathrm{F}$ is transferable only if it is possible that $\mathrm{F}$ be had by some substance other than $a$. Given DP, this means that $\mathrm{F}$ is transferable only if it is possible that $\mathrm{F}$ exist and $a \neq \mathrm{F}$. But again, identity is necessary, so because $a=\mathrm{F}$, it is impossible that $a \neq \mathrm{F}$. Thus, DP entails that principal attributes are not transferable. Thus, DP makes intersubstitutability impossible.

Independently of DP, IC provides a second block on the appeal to intersubstitutability. That is, even if we assume that DP is false, IC still precludes intersubstitutability. As we've seen, a pair of tropes are intersubstitutable only if each is transferable and neither is token dependent on its bearer. In this case, a trope requires only that it be borne by some substance or other and there is no token entity besides God that a trope requires to exist. Thus, if a principal attribute $F_{1}$ is transferable, then there is no entity besides God that $\mathrm{F}_{1}$ requires to exist. But if that is the case, then, according to $\mathrm{IC}, \mathrm{F}_{1}$ is a substance. More generally: if principal attributes are transferable, then they depend on their substances in the same way that their substances are said to depend on their modes. In each case, the sort of dependence is not token dependence. Thus, by parity of reasoning, if principal attributes are transferable, then IC will also count them as substances. But unless we identify principal attributes with their bearers (i.e., unless DP is true), it seems unacceptable to categorize principal attributes as substances. Thus, even if we assume that DP is false (and that IC doesn't entail DP), IC requires principal attributes to be non-transferable. Thus, independently of DP, IC rules out intersubstitutable principal attributes.

So a proponent of the separability argument cannot take $\mathrm{R}$ to be intersubstitutability. However, next to identity, taking $\mathrm{R}$ to be intersubstitutability would yield the strongest instances of S1 and S2. Thus, the unavailability of intersubstitutability would seem to lower the highest possible strength that the generalizing step could have.

As a third candidate for $\mathrm{R}$, consider falling under the same predicate. In the preceding, we've used similar names or labels for the principal attributes of actual minds as well as for merely possible minds. There is the "thought" trope of this mind, the "thought" trope of that mind, etc.; and it is possible that there be any number of "thought" tropes. But the mere similarity of these linguistic items isn't a good reason to assume that the tropes thus named would be exactly similar with respect to their incompossibility profiles - that is, with respect to the tropes with which they are or are not compossible. This is because however exactly similar these linguistic items are, their similarity doesn't strongly suggest, much less require, the exact similarity of the tropes to which they refer. Similarity comes in degrees, and as Wittgenstein famously pointed out, there are groups of greatly 
or even wholly dissimilar things that nonetheless all count as "games" (1953, $\S \S 66-67)$. So falling under the same predicate isn't a promising candidate for $\mathrm{R}$ because it doesn't guarantee exact similarity. The natural place to look next is to exact similarity itself.

As a fourth candidate for R, consider exact similarity. Recall that there are two general strategies for providing the generalizing step, and that the success of either strategy requires, as a first move, a defense of the claim that for any thought trope $\mathrm{T}^{*}$ and any extension trope $\mathrm{E}^{*}$, it is plausible that $\mathrm{T}_{1}$ and $\mathrm{T}^{*}$ stand in $\mathrm{R}$, and $\mathrm{E}_{1}$ and $\mathrm{E}^{*}$ stand in $\mathrm{R}$. Thus, exactly similarity will suffice for R only if (i) there is no thought trope that only inexactly resembles $\mathrm{T}_{1}$, and (ii) there is no extension trope that only inexactly resembles $\mathrm{E}_{1}$. Unfortunately, it is hard to see how a proponent of the separability argument can rule out the possibility that there are less than exactly similar thought (extension) tropes. Plausibly, for all we know, there could very well be such tropes. ${ }^{36}$ Similarity comes in degrees, falling under the same predicate doesn't entail being exactly similar, and significantly dissimilar items can satisfy the same predicate.

This suggests that if a champion of the tropist separability argument appeals to exact similarity, she has the burden of proof in ruling out the possibility that two thought (or extension) tropes are only inexactly similar. These points further suggest that this is a significant burden of proof.

Before moving to the second and final challenge, I want to briefly clarify the upshot of the first challenge. Of interest here is the general thesis that IC comes to the aid of the separability argument, enabling it to secure not only property dualism but at least local substance dualism. The upshot of the first challenge is that IC is a poisoned chalice. If principal attributes are universals, then IC aids the separability argument in establishing not only substance dualism but hyper substance dualism. A commitment to the latter can be avoided by taking principal attributes to be tropes rather than universals, but doing so requires the separability argument to have an additional non-trivial generalizing step (either $\mathrm{C} 1$ or C2). All things being equal, the addition of this step weakens the argument. And, because IC (directly or through DP) blocks the intersubstitutability and transferability of principal attributes qua tropes, IC makes the additional step more difficult to support. This is important because a proponent of the tropist separability argument would seem to have the burden of proof with respect to the generalizing step. In the absence of non-circular reasons for accepting S1 or S2, a person without a prior commitment to local substance dualism would seem to be within her rights to withhold on $\mathrm{C} 1$ and $\mathrm{C} 2$.

\section{SECOND CHALLENGE: SECURING DP}

Previously, I maintained that a joint commitment to IC and the assumption that principal attributes are universals is problematic in that it leads to HSD. In this section I will argue that a different problem arises from a joint commitment to IC and the assumption that principal attributes are tropes. In rough outline, I will argue that the problem is the following: The assumption that principle attributes are tropes is 
consistent with substances not being token-dependent on their principle attributes. That is, the assumption does not require a substance to be token-dependent on its principle attribute. But if that is true, then it is consistent with IC that a substance and its principal attribute are non-identical. Thus, if principal attributes are tropes, then IC does not secure DP. But if IC does not secure DP, then IC cannot play the crucial role of rescuing the separability argument from Regius's Objection. That is, if IC doesn't establish DP, then IC does not provide the argument with the premise needed to secure substance dualism and not merely property dualism. Furthermore, without DP a tropist separability argument does not even secure minimal substance dualism (and so the question of a generalizing step is moot).

To show this, it will be helpful to clarify why DP follows from a joint commitment to IC and the assumption that principal attributes are universals. If principal attributes are universals, then, necessarily, there is at most one attribute of extension. Thus, if any substance is extended, then there is a principal attribute of extension $\mathrm{E}$ and for any substance $x, x$ is extended only if $x$ has E. In this case, every essentially extended substance is token dependent on E, its principal attribute. But according to IC, God is the only distinct entity on which a substance can be token dependent. In other words, if substance $a$ is token dependent on some entity $b$, then either $a=b$ or $\operatorname{God}=b$. Thus, (on pain of identifying each principal attribute with God), a principal attribute is only conceptually distinct from its bearer (DP). In this way, the assumption that principal attributes are universals, when conjoined with IC, entails DP (and HSD, as we've seen above).

However, if principal attributes are tropes, then DP does not follow from IC. The inference from IC to DP requires, at the very least, the further assumption that tropes are non-replaceable. Where substance $a$ has trope F, F is non-replaceable if it is impossible that there be a distinct trope $\mathrm{F}^{*}$ such that $\mathrm{F}$ and $\mathrm{F}^{*}$ are exactly similar and $a$ has $\mathrm{F}^{*}$ rather than $\mathrm{F}$. But the assumption that tropes are non-replaceable is both substantive and of questionable tenability. It is substantive because nothing in the notion of a trope - a non-shareable and non-unique property-requires a trope to be non-replaceable. Moreover, non-replaceability is a dubious assumption. Qua non-unique, any trope $\mathrm{F}$ is such that, possibly, there is a distinct $\mathrm{F}^{*}$ such that $\mathrm{F}$ and $\mathrm{F}^{*}$ are exactly similar. That is, tropes are exactly duplicable. Given that they are duplicable, it would be surprising if tropes were non-replaceable. If anything, then, we should expect tropes to be replaceable, given that they are non-unique. However, for the point at hand, we don't need to assume that non-uniqueness entails replaceability. Rather, it is enough that the conjunction of tropism and IC fails to entail non-replaceability.

We can now see why the joint commitment to tropes and IC is not sufficient to secure DP. To wit: without the further and questionable assumption that tropes are non-replaceable, an extended substance does not require any token or specific principal attribute of extension, but only some principal attribute of extension or other. Thus, a substance could have had a principal attribute that is numerically distinct from the one it has. But if that is possible, then a substance is not token dependent on its principal attribute. But unless a substance is token dependent on its principal attribute-unless its principle attribute is non-replaceable — it is 
consistent with IC that a substance and its principal attribute be non-identical. In sum, when conjoined with the assumption that principal attributes are tropes, IC does not entail DP, and so does not secure DP*- the crucial premise of an IC-based separability argument.

We can also arrive at this conclusion by noting that unless tropes are nonreplaceable, they will be relevantly on par with modes. Recall that IC doesn't require that there be only a conceptual distinction (i.e., identity) between a substance and its modes. This is because modes are replaceable. For example, an extended substance does not have to have some specific or token mode of extension, but only some mode or other. For any given mode $\mathbf{M}$ that a substance has, it is possible that the substance have some mode that is distinct from M. Importantly, this is the case even with respect to exactly similar modes. In order to be spherical, an extended substance must have only some sphericity mode or other-there is no token or specific sphericity mode that the substance must have. With respect to sphericity modes, a spherical substance must have exactly one but no exact one. Where substance $a$ is spherical in virtue of having sphericity mode M, it is possible that $a$ be spherical in virtue of having sphericity mode $\mathbf{M}^{*}$ instead, where $\mathrm{M}$ and $\mathrm{M}^{*}$ are non-identical but exactly similar. Thus, because modes are replaceable, a substance is not token dependent on its modes. This is why it is consistent with IC that a substance and its modes be non-identical. Now, for the sake of comparison, assume what seems not only possible but plausible- that tropes are replaceable. If so, then a substance would depend on its principal attribute in the same way that it depends on its modes. An extended substance would not require any token principal attribute of extension but only one of the many possible principal attributes of extension. In other words, it would be the case that with respect to principal attributes of extension, an extended substance must have exactly one but no exact one. Thus, by parity of reasoning, if it is consistent with IC that a substance and its modes are non-identical, then it would be consistent with IC that a substance and its principal attribute are non-identical. The point of this comparison is this. Without the additional and doubtful assumption that tropes are non-replaceable, principle attributes (qua tropes) will be similar to modes in that IC will be consistent with the non-identity of a substance and its principle attribute. Thus, a joint commitment to tropes and IC is not sufficient to secure DP.

The upshot of the second challenge is the following. If principal attributes are tropes, then IC does not secure DP* (by way of DP). But without DP*, the argument fails to establish even the weaker, minimal version of substance dualism.

To close this section I want to offer two clarificatory comments about the second challenge. First, it is important to note that I am not arguing that there is something conceptually incoherent about the idea of a substance being token dependent on a trope. Rather, the point is this. Unlike the concept of a universal, there is nothing in the concept of a trope that requires a substance to be token dependent on one. Thus, the mere conjunction of IC and the assumption that principal attributes are tropes does not entail that a substance is token dependent on its principal attribute. And, consequently, the conjunction does not secure DP*-the crucial premise in the IC-based separability argument. 
Second, in raising the second challenge I have noted that for any given trope $\mathrm{F}$ it is possible that there be a distinct trope that is maximally similar to $\mathrm{F}$. This is consistent with the central argument of the first challenge. My claim there was that if exact similarity is to play the role of $\mathrm{R}$, then the proponent of the tropist separability argument needs to show (or provide reasons to believe) that there are no thought (extension) tropes that are less than exactly similar to $T_{1}\left(E_{1}\right)$. But the fact that there could be pairs of thought tropes that are exactly similar does not entail (or even suggest) that all thought tropes are exactly similar (much less that all possible thought tropes are exactly similar).

\section{CONCLUSION}

In this paper I've attempted to clarify the relative importance of IC in Descartes's metaphysics. I've done so by focusing on the thesis that IC plays a crucial role in Descartes's separability argument. I've argued that the argument faces serious challenges on two ways of thinking about the ontological status of principal attributes.

On the one hand, a joint commitment to IC and the assumption that principal attributes are universals is problematic in that it makes the separability argument too strong - it secures not just substance dualism but hyper substance dualism. This joint commitment does succeed in securing the crucial doctrine that a substance and its principal attribute are not distinct entities (DP). But the latter doctrine entails that it is possible that there is more than one mind (body) if and only if it is possible that there is more than one principal attribute of thought (extension). Unfortunately, however, if principal attributes are universals, then it is not possible that there be more than one attribute of thought (extension). We thus arrive at HSD.

On the other hand, a joint commitment to IC and the assumption that principal attributes are tropes is problematic in that it makes the separability argument doubly weak. According to the first challenge, the assumption that principal attributes are tropes avoids HSD but saddles the tropist argument with an additional and weakening generalizing step - a step that IC undermines. Without the generalizing step, the tropist argument fails to secure local substance dualism. And according to the second challenge, the assumption that principle attributes are tropes is consistent with a substance not being token dependent on its principle attribute. If anything, tropism suggests that a substance is not token dependent on its principle attribute. Thus, the conjunction of tropism and IC isn't sufficient to secure DP, and so not $\mathrm{DP}^{*}$, the crucial premise of an IC-based separability argument. Without DP*, the argument fails to secure even minimal substance dualism.

In sum, on either way of thinking about the ontological status of principal attributes, with respect to its role in a separability argument, IC is a poisoned chalice.

As a final thought, I want to offer a tentative reply to the following question: Supposing we could find a way other than IC to secure DP* for the tropist separability argument, would establishing mere minimal substance dualism suffice to secure one or more of Descartes's aims?

I think we can answer this in the affirmative. One of Descartes's explicit aims in the Meditations is to make a philosophical case for the immortality of the soul. 
The separability argument appears to be a crucial part of this case. However, given its essentially first-person style, it would seem more apt to say that the argument is supposed to provide a meditator with a potential way to discover his or her own immortality. ${ }^{37}$ And for this purpose, a separability argument for minimal substance dualism would seem to suffice. For consider: In order to determine whether $I$ am capable of immortality, I do not need to settle the question of whether or not all persons (much less all possible persons) are capable of immortality. If I can affirm the premises (i.e., as indexed to myself) of a valid separability argument, then I can conclude that I am a substance that is mental but not possibly extended. I would thereby discover something important (though perhaps not decisive) ${ }^{38}$ for settling the question of my own immortality. In other words, to the extent to which a separability argument is a crucial part of the case for one's own immortality, an individualized argument for minimal substance dualism will suffice. For this modest but important aim, an argument for a stronger form of dualism-whether local or global-is unnecessary, and so perhaps the first challenge can be set aside. This leaves the matter of whether or not a tropist separability argument for minimal dualism can overcome the second challenge - securing DP* on grounds other than IC. I leave this question for another day. ${ }^{39}$

\section{ENDNOTES}

1. Some scholars have rejected this traditional view, arguing instead that Descartes was a substance trialist. On this interpretation, a human being is a substantial union of mind and body, where this union (the human being) is itself a third kind of substance. I have nothing directly to say about trialism in what follows. For a defense of trialism, see the work by Paul Hoffman, especially his 1986 and 1999. For further references and an extended critique, see Kaufman 2008.

2. For discussion see Bennett 2001; Garber 1992 (who calls it the "complete concept argument”); Garrett 2009; Hooker 1978; Kaufman 2008; Kenny 1968; Rodriguez-Pereyra 2008; Rozemond 1998; Schiffer 1976; Williams 1978; and Wilson 1978 (who calls it the “epistemological argument”).

3. See Loeb 1981; Bennett 2001; and Rodriguez-Pereyra 2008.

4. References are to be understood as follows: 'CSM' stands for The Philosophical Writings of Descartes, vols. 1-2, trans. J. Cottingham, R. Stoothoff, and D. Murdoch (Cambridge: Cambridge university Press, 1985) [Descartes 1985]. 'CSMK' stands for The Philosophical Writings of Descartes, vol. 3, trans. J. Cottingham, R. Stoothoff, D. Murdoch, and A. Kenny (Cambridge: Cambridge university Press, 1991) [Descartes 1991]. 'AT' stands for Adam and Tannery's edition of Descartes's works (Paris: Vrin/C.N.R.S., 1964-1976) [Descartes 1964].

5. In Proposition IV of the Second Replies, Descartes presents a generalized version of the argument, speaking of "the mind, that is, a thinking substance" and "the body, that is, ... an extended substance" (AT VII, 170).

6. For example, see Garrett 2009, 87n6. 
7. Garrett $(2009,87 \mathrm{n} 6)$ writes: "Although its stated conclusion and some of its premises are restricted to Descartes' own mind and body, the argument may be readily generalized to conclude that every mind is distinct from every body, simply by replacing his references to his own mind and body with references to all minds and bodies, respectively." RodriguezPereyra $(2008,72)$ says that in the Sixth Meditation "Descartes puts the argument in the first person, i.e., in terms of himself and his body. But this is not essential to the argument." It is, thus, interesting to find the following admission from Bennett $(2001,71)$ : "I have nothing useful to say about the generalizing move from 'I am immaterial' to 'All thinking things are immaterial' or 'No matter thinks'.' For a different view, see Secada 2000, 253-255.

8. Others who do so include Garber 1992, 85-89; Rodriguez-Pereyra 2008, 73; and Yablo 1990.

9. For more discussion see Rodriguez-Pereyra 2008, 73.

10. As Michael LeBuffe pointed out to me, it is not entirely clear whether Descartes ever claims to clearly and distinctly conceive his body. For my purposes, this inclusion is innocent: in so far as I raise problems for the separability argument, these problems would not be alleviated were we to withhold the premise in question.

11. This is an amplified paraphrase of what Regius says in his broadsheet against Descartes (CSM I, 294-295; AT VIIB, 343).

12. The more general conclusion is arrived at as follows. Something has mental properties (e.g., modes of thought) if and only if it has a principal attribute of thought. And, something has material properties (e.g., modes of extension) if and only if it has a principal attribute of extension. Thus, given (6), it follows that (a) mental properties can be had by something that lacks material properties, and (b) material properties can be had by something that lacks mental properties. Thus, by (7), mental properties and material properties are non-identical.

13. I take this wording from Rodriguez-Pereyra 2008, 70.

14. This construal of IC leaves an important question unanswered-namely, whether God's existence could require some type of distinct entity or other. I have nothing to say about this question in what follows.

15. Rodriguez-Pereyra doesn't consider this option, but it suggests that the inference from IC to DP isn't as straightforward as he suggests. For a related discussion, see Schmaltz 1991.

16. According to Rodriguez-Pereyra, IC's importance for Descartes's metaphysics is also evinced by the fact that because IC uniquely secures DP, it thereby secures a direct entailment of DP, the Cartesian principle that a substance has exactly one principal attribute.

17. For a discussion of the necessity of identity and its relevance for Descartes's separability argument, see Bennett 2001, 76-81.

18. The general conclusion is arrived at via the following suppressed premise: A substance has a mental property (e.g., modes of thought) only if it has a principal attribute of thought, and a substance has a material property (e.g., modes of extension) only if it has a principal attribute of extension. The latter, together with (11), entail that no substance has both a mental property and a material property.

19. Important contemporary expositions of trope theory include Williams 1953; Campbell 1990; Maurin 2002; and Ehring 2011.

20. Whether the parenthetical qualifier ("at once") is necessary depends on whether a trope is transferable, which is a matter of dispute among trope theorists. More on this below. 
21. Nolan (1998) argues that Descartes did not accept universals. For the thesis that Descartes's principal attributes are trope-like, see Nolan 1997 and Rodriguez-Pereyra 2008, $78 \mathrm{n} 15$.

22. It is tempting to describe these as haecceitistic universals, but while that would capture the idea that they are unique and non-sharable, it would also misleadingly suggest that they are not qualitative.

23. One way to put this is to say that unique properties are subject to an intraworld identity of indiscernibles: Necessarily, for any property $F$, if there is a property $\mathrm{G}$ such that $\mathrm{F}$ and $\mathrm{G}$ are exactly similar, then $\mathrm{F}=\mathrm{G}$. Whether and in what sense a universal is supposed to be unique is a neglected issue among realists, who typically emphasize shareability. But shareability does not entail uniqueness. Indeed, there isn't any obvious incoherence in the idea that there are, say, seven shareable properties of sphericity, each with its own set of instances. Nevertheless, in what follows I assume that universals are unique. I do so for two reasons. First, the inference from shareability to uniqueness seems well motivated — on grounds of parsimony, for example. The next two reasons are more important, as they concern the aims of this paper. Second, as noted above, DP entails that a principle attribute is non-shareable. Thus, taking principle attributes to be shareable is not a viable way to understand a separability argument that takes DP as a premise. This leaves two options to consider: non-sharable and unique properties (hybrid-universals) and non-sharable and non-unique properties (tropes). Third, the implications of DP notwithstanding, if we took principle attributes to be non-unique and shareable properties, then the separability argument would require a generalizing step. As such, it would face similar challenges as those I pose below for the tropist argument. I thank Rodriguez-Pereyra and an anonymous referee for pressing me on the sense in which universals are supposed to be unique.

24. For discussion see Hoffman 1986; Palmer 2002; Sowaal 2004; and Lennon 2007.

25. John Hawthorne (2007, 90n6) notes that "on some of Descartes's uses, [modes are] trope-like instances of determinates."

26. In a letter to an unknown correspondent, Descartes writes: "Thus shape and motion are modes, in the strict sense, of corporeal substance; because the same body can exist at one time with one shape and at another with another, now in motion and now at rest; whereas, conversely, neither this shape nor this motion can exist without this body." (CSMK, 280; AT IV, 349) Cited by Rodriguez-Pereyra 2008, 81.

27. For example, see Garrett 2009, 87n6.

28. In his discussion of Descartes's separability argument, Rodriguez-Pereyra presents a definition of dualism that lacks the modal qualifier 'necessary.' I've learned through correspondence that this was intentional on his part, as he takes Descartes's separability argument to aim at only (what I'm calling) a local version of dualism.

29. I am also setting aside the interesting question of whether and how LSD could be true while GSD is false.

30. It might be tempting to construe the minimalist version as follows, but it won't do: There is a principal attribute of thought $\mathrm{T}$ and an principal attribute of extension $\mathrm{E}$, and no substance has both $\mathrm{T}$ and $\mathrm{E}$. Unfortunately, this is consistent with there being an attribute of extension $\mathrm{E}^{*}$ such that some substance has both $\mathrm{T}$ and $\mathrm{E}^{*}$. Thus, if principal attributes are tropes, characterizing even a minimalist version of dualism requires a quantifier that ranges over all principal attributes of extension and thought.

31. cf. n5. 


\section{But see the comment in $n 10$.}

33. It is important not to confuse intersubstitutability with intrasubstitutability. The latter concerns the substitutabilty of two tropes with respect to the very same substance. For any substance $a$ that has trope $\mathrm{F}$, if it is possible that $a$ have some distinct trope $\mathrm{F}^{*}$ (rather than $\mathrm{F}$ ) without resulting in a non-trivial change to $a$, then $\mathrm{F}_{\text {and }} \mathrm{F}^{*}$ are intrasubstitutable. Our present concern is with the defense of $\mathrm{S} 1$ or $\mathrm{S} 2$, and these require a candidate for $\mathrm{R}$ that can hold between $\mathrm{T}_{1}$ (or $\mathrm{E}_{1}$ ) and any possible thought (or extension) trope, and not just any two tropes that a given substance could have. So intrasubstitutability isn't an interesting candidate for $\mathrm{R}$.

34. For example, suppose the following: substance $a$ has sphericity trope $S_{1}, S_{1}$ is token dependent on $a$, and $\mathrm{S}_{1}$ is transferable. It is consistent with that assumption that $\mathrm{S}_{1}$ belong to some other substance $b$. However, even if $\mathrm{S}_{1}$ were to belong to $b, \mathrm{~S}_{1}$ would cease to exist if $a$ ceased to exist. In this case, we get the strange result that $b$ 's being what it is is dependent on the existence of something besides $b$ and $\mathrm{S}_{1}$. Indeed, if $a$ ceases to be, then either (i) $b$ ceases to be, (ii) $b$ continues to be but is no longer spherical, or (iii) $b$ continues to be and remains spherical. Each option leads to arguably unacceptable results. If (i), then substances can depend on each other for their existence in a rather arbitrary way. For example, $b$ could have had $a$ 's sphericity trope, and, if that had been the case, then $b$ would have existed only if $a$ did. In other words, $b$ doesn't actually existentially depend on $a$, but $b$ could have so depended. If (ii), then substances can depend on each other for their character in a rather arbitrary way. For example, the passing of $a$ would change the shape of $b$. On this assumption, the passing of one substance $(a)$ would change the shape of another $(b)$. So $b$ 's being spherical is dependent on the existence of $a$. This might be acceptable for some pairs of substances, but it is unacceptable as a general implication of the joint assumption that tropes are transferable and token dependent. If (iii), then the annihilation of $a$ would not only bring about the destruction of one of $b$ 's tropes, it would also bring about the de novo creation of a trope to replace the one it destroyed. In this case, the passing of $a$ would not change the truth value of " $b$ is spherical." However, with respect to before and after the passing of $a$, $b$ would be spherical in virtue of having distinct sphericity tropes. Thus, (iii) has the unacceptable implication that the destruction of one substance could bring about a qualitatively indiscernible trope-replacement in another. Thus, token dependence plus transferability leads to unacceptable results. If a trope is transferable, then it is implausible that it is token dependent on its bearer.

35. A couple points of clarification: transferability and being had contingently are logically independent of each other. For example, non-transferability is consistent with being had contingently. Non-transferability means that the trope cannot have a bearer besides the one it has. It does not mean that the bearer must have that specific (token) trope. Thus, a nontransferable trope can be had contingently. In addition, the fact that two tropes are each transferable does not entail that they are intersubstitutable. The latter requires the further (3rd) condition that they be swappable without non-trivally changing their bearers.

36. This is true even on the plausible assumption that Descartes's principle attributes should be understood as determinable tropes. Not every determinable is a highest-level determinable. And, two less-than-highest-level determinables can fall under the same highest-level determinable but be dissimilar. For example, redness and blueness are determinable color tropes (determined by, say, scarlet and navy, respectively) but not highest-level determinables (each falls under the highest-level determinable coloredness). Although redness and blueness are determinables, they are not exactly similar. Thus, two color tropes can be determinables without being exactly similar. For similar reasons, it is possible that two principle attributes 
of thought are determinables without being exactly similar. It is possible (and arguably not unlikely, given the existence of different kinds of minds, such as human, divine, and angelic) that the meditator's principle attribute of thought is a less-than-highest-level determinable. In other words, for all I know (qua meditator), my principle attribute of thought is on par with redness (it is a determinable but not a highest-level determinable) and possibly, there is a distinct attribute of thought on par with blueness (a determinable that is dissimilar to my principle attribute of thought). In sum, if exact-similarity is to play the role of $\mathrm{R}$ (and so accomplish the generalizing step), then a meditator needs to rule out the possibility that two thought tropes are only inexactly similar. To do this, she needs to rule out the possibility that her thought trope is a less-than-highest-level determinable trope. It is not obvious that she is a position to rule this out. For a very similar line of argument, see Jorge Secada 2000, 253-255.

\section{Cf. Secada 2000, 255.}

38. As Father Mersenne pointed out, immortality does not immediately follow from the fact that the soul is independent from the body (CSM II, 91). Descartes conceded the point but argued that we have no reason to think that something like bodily death could destroy a mental substance (CSM II, 109).

39. For numerous valuable comments and suggestions, I am especially grateful to Gonzalo Rodriguez-Pereyra, Michael LeBuffe, Stephen Daniel, and the anonymous referees of the Journal of Philosophical Research. For helpful discussion, I also wish to thank José Tomás Alvarado Marambio, Todd Buras, Don Garrett, Dan Kaufman, Nathan King, Michael Loux, Chris Menzel, J. P. Moreland, Alan Nelson, Timothy Pickavance, Marleen Rozemond, and the audience at the 3rd Coloquio Internacional, Sociedad Chilena de Filosofía Analítica, Biblioteca Nacional, Santiago, Chile, May 13, 2011.

\section{BIBLIOGRAPHY}

Bennett, Jonathan. 2001. Learning from Six Philosophers: Descartes, Spinoza, Leibniz, Locke, Berkeley, Hume, Volume 1. Oxford: Oxford University Press.

Campbell, Keith. 1990. Abstract Particulars. Oxford: Basil Blackwell.

Descartes, Rene. 1964. Oeuvres de Descartes, Volumes 1-12, ed. C. Adam and P. Tannery. Paris: J. Vrin.

1985. The Philosophical Writings of Descartes, Volumes 1-2, trans. John Cottingham, Robert Stoothoff, and Dugald Murdoch. Cambridge: Cambridge University Press. 1991. The Philosophical Writings of Descartes, Volume 3, trans. J. Cottingham, R. Stoothoff, D. Murdoch, and A. Kenny. Cambridge: Cambridge University Press.

Ehring, Douglas. 2011. Tropes: Properties, Objects, and Mental Causation. Oxford: Oxford University Press.

Garber, Daniel. 1992. Descartes' Metaphysical Physics. Chicago: University of Chicago Press.

Garrett, Daniel. 2009. "Descartes, Spinoza, and Locke on Extended Thinking Beings.” In Topics in Early Modern Philosophy of Mind, ed. Jon Miller, 85-104. Studies in the History of Philosophy of Mind, vol. 9. Dordrecht: Springer Press.

Hawthorne, John. 2007. “Cartesian Dualism.” In Persons: Human and Divine, ed. Peter van Inwagen and Dean Zimmerman, 87-98. Oxford: Oxford University Press. 
Hoffman, Paul. 1986. "The Unity of Descartes's Man.” The Philosophical Review 95: 339-370. doi: http://dx.doi.org/10.2307/2185464 1999. "Cartesian Composites." Journal of the History of Philosophy 37: 251-270. doi: http://dx.doi.org/10.1353/hph.2008.0912

Hooker, Michael. 1978. “Descartes's Denial of Mind-Body Identity.” In Descartes: Critical and Interpretive Essays, ed. Michael Hooker, 171-185. Baltimore, MD: Johns Hopkins University Press.

Kaufman, Dan. 2008. "Descartes on Composites, Incomplete Substances, and Kinds of Unity." Archiv für Geschichte der Philosophie 90: 39-73. doi: http://dx.doi.org/10.1515/AGPH.2008.002

Kenny, Anthony. 1968. Descartes. New York: Random House.

Lennon, Thomas. 2007. "The Eleatic Descartes." Journal of the History of Philosophy 45: 29-47. doi: http://dx.doi.org/10.1353/hph.2007.0013

Loeb, Louis. 1981. From Descartes to Hume: Continental Metaphysics and the Development of Modern Philosophy. New York: Cornell University Press.

Maurin, Anna-Sofia. 2002. If Tropes. Dordrecht: Kluwer Academic Publishers. doi: http://dx.doi.org/10.1007/978-94-017-0079-5

Nolan, Lawrence. 1997. "Reductionism and Nominalism in Descartes's Theory of Attributes." Topoi 16: 129-140. doi: http://dx.doi.org/10.1023/A:1005899729236

_. 1998. "Descartes' Theory of Universals.” Philosophical Studies 89: 161-180. doi: http://dx.doi.org/10.1023/A:1004228828029

Palmer, Eric. 2002. "Descartes on Nothing in Particular." In New Essays on the Rationalists, ed. Rocco Gennaro and Charles Huenemann, 26-47. New York and Oxford: Oxford University Press.

Rodriguez-Pereyra, Gonzalo. 2008. "Descartes's Substance Dualism and His Independence Conception of Substance." Journal of the History of Philosophy 46: 69-90. doi: http://dx.doi.org/10.1353/hph.2008.1827

Rozemond, Marleen. 1998. Descartes's Dualism. Cambridge, MA: Harvard University Press.

Schiffer, Stephen. 1976. "Descartes on His Essence.” The Philosophical Review 85: 21-43. doi: http://dx.doi.org/10.2307/2184253

Schmaltz, Tad. 1991. "Platonism and Descartes' View of Immutable Essences." Archiv für Geschichte der Philosophie 73: 129-170.

doi: http://dx.doi.org/10.1515/agph.1991.73.2.129

Secada, Jorge. 2000. Cartesian Metaphysics: The Scholastic Origins of Modern Philosophy. New York: Cambridge University Press.

doi: http://dx.doi.org/10.1017/CBO9780511487309

Sowaal, Alice. 2004. "Cartesian Bodies." Canadian Journal of Philosophy 34: 217-240.

Williams, Bernard. 1978. Descartes. New York: Penguin.

Williams, D. C. 1953. “On the Elements of Being: I.” The Review of Metaphysics 7: 3-18.

Wilson, Margaret. 1978. Descartes. London: Routledge and Kegan Paul. doi: http://dx.doi.org/10.4324/9780203167670

Wittgenstein, Ludwig. 1953. Philosophical Investigations. New York: Macmillan Publishing Co.

Yablo, Stephen. 1990. "The Real Distinction Between Mind and Body.” Canadian Journal of Philosophy 16: 149-201. 